\title{
Red blood cell distribution width is associated with adverse cardiovascular events after septal myectomy
}

\author{
Shengwei Wang ${ }^{1}$, Changpeng Song ${ }^{2}$, Hao Cui ${ }^{3}$, Changsheng Zhu ${ }^{4}$, Rong Wu ${ }^{4}$, Xiaohong Huang ${ }^{2}$, \\ Yongqiang Lai ${ }^{1}$, Shuiyun Wang ${ }^{4}$ \\ ${ }^{1}$ Department of Cardiovascular Surgery Center, Beijing Anzhen Hospital, Capital Medical University, Beijing Institute of Heart, Lung and Blood \\ Vascular Diseases, Beijing, China; ${ }^{2}$ Department of Special Medical Treatment Center, State Key Laboratory of Cardiovascular Disease, Fuwai \\ Hospital, National Center for Cardiovascular Diseases, Chinese Academy of Medical Sciences and Peking Union Medical College, Beijing, China; \\ ${ }^{3}$ Department of Cardiovascular Surgery, Mayo Clinic, Rochester, Minnesota, USA; ${ }^{4}$ Department of Cardiovascular Surgery, State Key Laboratory \\ of Cardiovascular Disease, Fuwai Hospital, National Center for Cardiovascular Diseases, Chinese Academy of Medical Sciences and Peking Union \\ Medical College, Beijing, China \\ Contributions: (I) Conception and design: S Wang; (II) Administrative support: S Wang; Y Lai; (III) Provision of study materials or patients: S Wang; \\ (IV) Collection and assembly of data: S Wang, C Song, H Cui, C Zhu, R Wu; (V) Data analysis and interpretation: S Wang, C Song, X Huang; (VI) \\ Manuscript writing: All authors; (VII) Final approval of manuscript: All authors. \\ Correspondence to: Shuiyun Wang, MD. No. 167, Beilishi Road, Xicheng District, Beijing 100037, China. Email: wsymd@sina.com; Yongqiang Lai, \\ MD. No. 2, Anzhen Road, Chaoyang District, Beijing 100029, China. Email: lyq_md@126.com.
}

Background: Red blood cell distribution width (RDW) is associated with increased morbidity and mortality in several cardiovascular diseases. However, the prognostic significance of RDW in patients with hypertrophic obstructive cardiomyopathy (HOCM) who underwent septal myectomy remains unclear as no studies have been conducted on this topic. This study aimed to assess the prognostic significance of RDW in these patients.

Methods: A total of 867 adults with HOCM who underwent septal myectomy at Fuwai Hospital from 2011 to 2017 were retrospectively studied. All patients were assessed comprehensively, including their medical history, echocardiograms, and blood test results.

Results: The median age of patients was 47.9 [interquartile range (IQR), 37.0-56.0] years and 61.5\% of patients were men. During a median follow-up period of 32 (IQR, 17-53) months, 26 patients died and 23 had a cardiovascular death during follow-up. Compared to patients in the lowest RDW quartile, those in the highest quartile had a significantly lower 5-year survival free from all-cause and cardiovascular death (95.9\% vs. $87.6 \%, \mathrm{P}<0.001 ; 95.9 \%$ vs. 89.9\%, $\mathrm{P}<0.001$ ). Compared with lower RDW, higher RDW was significantly associated with all-cause and cardiovascular death after adjustment for age, sex, body mass index, and relevant clinical risk factors [per RDW standard deviation (SD) hazard ratio (HR) increase $=1.76$, 95\% confidence interval (CI): 1.54-2.05, $\mathrm{P}<0.001$; per RDW SD HR =1.91, 95\% CI: 1.63-2.22, P for trend $<0.001]$.

Conclusions: Higher RDW is independently associated with all-cause and cardiovascular death in patients with HOCM after septal myectomy. Therefore, this readily available biomarker could be considered as an additive biomarker for risk stratification in these patients.

Keywords: Hypertrophic obstructive cardiomyopathy (HOCM); red blood cell distribution width (RDW); septal myectomy

Submitted Jul 25, 2020. Accepted for publication Nov 22, 2020.

doi: $10.21037 /$ jtd-20-2528

View this article at: http://dx.doi.org/10.21037/jtd-20-2528

(C) Journal of Thoracic Disease. All rights reserved. 


\section{Introduction}

Hypertrophic cardiomyopathy (HCM) is a common inherited heart disease with a prevalence of approximately 1:500 in the general population. Approximately two-thirds of patients with HCM have left ventricular outflow tract obstruction, which is considered hypertrophic obstructive cardiomyopathy (HOCM). When symptoms in these patients are refractory to optimal pharmacologic therapy, septal myectomy is recommended (1). Previous studies have reported that myocardial fibrosis, as measured using late gadolinium enhancement cardiovascular magnetic resonance, is an independent predictor of adverse outcomes in patients with HCM (2). However, considering the high cost and popularity of cardiac magnetic resonance, cheaper and more effective predictors are required.

Red blood cell distribution width (RDW) is a measurement parameter of the variability of the size of circulating red blood cells, and high RDW reflects an elevated reticulocyte level because of the possibility of iron deficiency, hemolytic anemia, or folate and vitamin B deficiency (3). Recently, many studies have reported that RDW is associated with morbidity and mortality in patients with heart failure $(4,5)$, coronary artery disease $(6,7)$, pulmonary hypertension (8), and those who have previously undergone transcatheter aortic valve implantation or coronary artery bypass surgery $(7,9,10)$. In addition, high RDW is associated with inflammatory, the incidence of atrial fibrillation, and so on (11). All of these had an adverse impact on the clinical outcomes for patients with HCM. Furthermore, a recent study also found that high RDW is an independent predictor of heart failure hospitalization in these patients (12). However, studies on the prognostic significance of RDW in patients who underwent septal myectomy are scant. Therefore, the present study was conducted to assess the prognostic significance of RDW in these patients.

We present the following article in accordance with the STROBE reporting checklist (available at http://dx.doi. org/10.21037/jtd-20-2528).

\section{Methods}

\section{Study population}

We retrospectively included 867 consecutive patients with HOCM evaluated at Fuwai Hospital in Beijing between 2011 and 2017. The diagnostic criteria of HOCM and surgical indications were consistent with the 2011 American
Heart Association/American College of Cardiology guidelines and 2014 European Society of Cardiology guidelines, which mainly include an unexplained septal hypertrophy with a thickness of $>15 \mathrm{~mm}(1,13)$. The indications for septal myectomy were (I) severe symptoms or syncope or near-syncope despite optimal medical therapy and (II) an LV outflow tract (LVOT) gradient $>50 \mathrm{mmHg}$ at rest or with provocation. Surgical approaches were detailed in our previous publication (14). Concomitant operations were appropriately performed based on preoperative evaluation and intraoperative exploration. Following discharge from the hospital, demographic and clinical data of all patients were collected. This study was approved by the Ethics Committee of Fuwai Hospital, Chinese Academy of Medical Sciences (Ethic committee study number: 2019-1213). Informed consent was obtained from all patients. The study was conducted in accordance with the tenets of the Declaration of Helsinki (as revised in 2013).

\section{Imaging analysis}

Echocardiography was performed on patients by an experienced physician using an E9 ultrasound system. Diameters of the cardiac chambers were expressed as the maximum value of the anteroposterior diameter in cardiac cycles. The thicknesses of the interventricular septum and ventricular wall were determined during diastole. Aside from the maximum thickness, the representative interventricular septal thickness was also recorded to indicate the overall thickness. The LVOT gradient was calculated using a simplified Bernoulli equation. Measurements of left ventricular ejection fraction were determined by following the American Society of Echocardiography recommendations.

\section{Laboratory measurements}

A fasting blood sample was obtained from all patients on the second day of hospitalization before surgery. Hemoglobin levels, mean corpuscular volume, RDW, mean platelet volume, platelet distribution width, and platelet count were measured on an automatic hematology analyzer (XT1800i, Sysmex, Japan) on samples obtained for standardof-care evaluation. The inter-run coefficient of variation of the RDW assay during the study period was routinely $1 \%$. Other laboratory parameters, including high-sensitivity C-reactive protein (hs-CRP), creatinine, and low-density and high-density lipoprotein, were also monitored 
concurrently. We estimated the glomerular filtration rate using the following equation: $\mathrm{a} \times(\text { serum creatinine } / \mathrm{b})^{\mathrm{c}}$ $\times(0.993)^{\text {age }} ; \mathrm{a}=144$ for women or 141 for men; $\mathrm{b}=0.7$ for women or 0.9 for men; $c=-0.329$ if the creatinine is $<0.7 \mathrm{mg} / \mathrm{dL}$ or -1.209 if the creatinine is $>0.7 \mathrm{mg} / \mathrm{dL}$ for women; $\mathrm{c}=-0.411$ if the creatinine is $<0.7 \mathrm{mg} / \mathrm{dL}$ or -1.209 if the creatinine is $>0.7 \mathrm{mg} / \mathrm{dL}$ for men.

\section{Endpoints and follow-up data}

The clinical status was obtained through telephonic interviews with patients or family members at least once a year after septal myectomy. Patients who died were censored the same day. The last follow-up of survivors was conducted in June 2018. Survival analysis included both allcause and cardiovascular mortality.

\section{Statistical analysis}

The Kolmogorov-Smirnov test was used to assess normal distribution of continuous variables. The results are expressed as mean \pm standard deviation (SD), median [interquartile range (IQR)], or percentage, as appropriate. We used $\chi^{2}$ or one-way ANOVA to test for differences in categorical or continuous factors between different categories of RDW, respectively. Multivariable linear regression was used to analyze factors associated with RDW. The Kaplan-Meier method was used to calculate survival free from the endpoint events. A log-rank test was used to compare survival curves among the different patient groups. Differences in baseline characteristics among the groups were adjusted using multivariate Cox models. Enter techniques were used to identify variables independently associated with the endpoints in these analyses that were incorporated into the final models. Variables with $\mathrm{P}<0.1$ on univariate analysis were entered into a multivariate analysis. All reported probability values were two-tailed, and a $\mathrm{P}$ value $<0.05$ was considered statistically significant. The SPSS version 24.0 statistical software (IBM) and GraphPad Prism 7.0 (GraphPad Software Inc., La Jolla, CA, USA) were used for calculations and illustrations, respectively.

\section{Results}

\section{Baseline patient characteristics}

We enrolled 867 patients with HOCM who underwent septal myectomy (533 men and 334 women). The median age of the entire population was 47.9 (IQR, 37.0-56.0) years. Baseline characteristics of subgroups based on the quartile of RDW are described in Table 1.

The median value of RDW in the overall population was $12.8 \%$ (IQR, 12.4-13.4\%). Patients with higher RDW tended to be older, were more likely to women, and had higher body mass index, as well as lower hemoglobin levels, low-density lipoprotein, and mean corpuscular volume. Importantly, the prevalence of syncope increases significantly with $\mathrm{RDW}(7.3 \%$ vs. $16.8 \%$ vs. $14.1 \%$ vs. $16.6 \%, \mathrm{P}=0.008)$. Other factors that were independently associated with baseline RDW are shown in Table 1 . In addition, RDW was significantly elevated in both patients who had an all-cause death and those who had a cardiovascular death compared with those who did not reach the endpoint $(13.03 \pm 1.18$ vs. $14.55 \pm 2.31$ vs. $14.67 \pm 2.42$, $\mathrm{P}<0.001)$ (Figure 1). Additional factors associated with the increase in RDW are shown in Table S1.

\section{Perioperative data among the different groups}

Perioperative data of subgroups according to the quartile of RDW are described in Table S2. Patients with higher RDW tended to have a higher proportion of concomitant Maze procedure and lower proportion of myocardial unroofing. In addition, the postoperative ventilation time and postoperative hospital stay tended to be longer in patients with a higher RDW. Nevertheless, no difference was found among the different groups in terms of other perioperative factors (Table S2).

\section{Association between RDW and clinical outcomes}

The mean follow-up period was $34.8 \pm 17.2$ months. During the follow-up period, a total of 26 patients died and 23 had a cardiovascular death. Of these deaths, 3 patients died of cancer, 13 died of sudden cardiac death, 1 died of infective endocarditis, 6 died of heart failure, and 1 died of myocardial infarction. In addition, 2 patients died during the perioperative period. The Kaplan-Meier curves showed that 5-year survival free from all-cause and cardiovascular death were significantly higher in those in the lowest quartile than in those in the highest quartile $(95.9 \% v s$. $96.3 \%$ vs. $95.0 \%$ vs. $87.6 \%, \mathrm{P}=0.003 ; 95.9 \%$ vs. $97.5 \%$ vs. $95.0 \%$ vs. $89.9 \%, \mathrm{P}=0.01$ ) (Figure 2 ).

Univariate and multivariate Cox analyses demonstrated that as a continuous variable, RDW was associated with an increased risk of all-cause and cardiovascular mortality 
Table 1 Baseline characteristics of patients, divided according to red blood cell distribution width

\begin{tabular}{|c|c|c|c|c|c|}
\hline Variable & \multicolumn{4}{|c|}{ RDW } & $P$ value \\
\hline \multicolumn{6}{|l|}{ Demographics } \\
\hline Age, years & $42.2 \pm 13.1$ & $46.9 \pm 13.7$ & $47.3 \pm 13.4$ & $47.3 \pm 14.2$ & $<0.001$ \\
\hline Male sex, \% & $173(70.3)$ & $122(60.4)$ & $151(68.6)$ & $87(43.7)$ & $<0.001$ \\
\hline Heart rate, beats/min & $73.2 \pm 9.8$ & $72.2 \pm 9.7$ & $73.9 \pm 9.4$ & $72.0 \pm 9.1$ & 0.139 \\
\hline NYHA class & $2.9 \pm 0.5$ & $2.8 \pm 0.5$ & $2.9 \pm 0.5$ & $2.9 \pm 0.5$ & 0.137 \\
\hline Family history, \% & $40(16.3)$ & $34(16.8)$ & $31(14.1)$ & $32(16.1)$ & 0.874 \\
\hline Course of disease, years & $4.0(2.0-8.0)$ & $5.0(1.0-8.0)$ & $5.0(2.0-9.0)$ & $5.0(2.0-9.0)$ & 0.450 \\
\hline Chest pain & $58(23.6)$ & $58(28.7)$ & $60(27.3)$ & $57(28.6)$ & 0.564 \\
\hline Palpitation & $31(12.6)$ & $20(9.9)$ & $25(11.4)$ & $31(15.6)$ & 0.355 \\
\hline Amaurosis & $21(8.5)$ & $16(7.9)$ & $24(10.9)$ & $15(7.5)$ & 0.607 \\
\hline Syncope & $18(7.3)$ & $34(16.8)$ & $31(14.1)$ & $33(16.6)$ & 0.008 \\
\hline Chest distress & $121(49.2)$ & $111(55.0)$ & $124(56.4)$ & $120(60.3)$ & 0.020 \\
\hline \multicolumn{6}{|l|}{ Comorbidities, n (\%) } \\
\hline Hypertension & $40(16.3)$ & 39 (19.3) & $59(26.8)$ & $43(21.6)$ & 0.040 \\
\hline Hyperlipemia & $24(9.8)$ & $25(12.4)$ & $32(14.5)$ & $29(14.6)$ & 0.355 \\
\hline LVEDD, mm & $41.5 \pm 5.2$ & $42.1 \pm 5.3$ & $42.4 \pm 5.4$ & $42.3 \pm 5.5$ & 0.244 \\
\hline IVST, mm & $20.6 \pm 5.7$ & $20.2 \pm 5.2$ & $20.6 \pm 5.7$ & $20.2 \pm 5.3$ & 0.824 \\
\hline Posterior LV wall, mm & $11.7 \pm 2.7$ & $11.9 \pm 2.7$ & $12.1 \pm 2.6$ & $12.3 \pm 2.7$ & 0.068 \\
\hline LVOT gradient, mmHg & $77.3 \pm 25.3$ & $81.5 \pm 29.4$ & $79.3 \pm 25.6$ & $86.1 \pm 28.1$ & 0.006 \\
\hline LVEF, \% & $71.3 \pm 6.6$ & $71.3 \pm 5.9$ & $71.9 \pm 6.5$ & $71.6 \pm 6.2$ & 0.755 \\
\hline Mitral regurgitation & $1.7 \pm 0.7$ & $1.9 \pm 0.7$ & $1.8 \pm 0.7$ & $2.0 \pm 0.6$ & 0.004 \\
\hline Pulmonary hypertension & $17(6.9)$ & $19(9.4)$ & $18(8.2)$ & $29(14.6)$ & 0.020 \\
\hline \multicolumn{6}{|l|}{ Medication, n (\%) } \\
\hline Beta-blockers & $175(71.1)$ & $145(71.8)$ & $168(76.4)$ & $147(73.9)$ & 0.592 \\
\hline Calcium-channel blockers & $23(9.3)$ & $17(8.4)$ & $18(8.2)$ & $18(9.0)$ & 0.969 \\
\hline
\end{tabular}

Table 1 (continued) 
Table 1 (continued)

\begin{tabular}{|c|c|c|c|c|c|}
\hline Variable & \multicolumn{4}{|c|}{ RDW } & $P$ value \\
\hline \multicolumn{6}{|l|}{ Laboratory variables } \\
\hline Log(NT-pro-BNP) & $2.99 \pm 0.50$ & $3.03 \pm 0.48$ & $3.11 \pm 0.47$ & $3.26 \pm 0.10$ & $<0.001$ \\
\hline Creatinine, $\mu \mathrm{mol} / \mathrm{L}$ & $76.6 \pm 14.7$ & $74.3 \pm 14.9$ & $77.9 \pm 14.9$ & $73.7 \pm 16.5$ & 0.015 \\
\hline Hs-CRP, mg/L & $0.9(0.4-1.5)$ & $1.0(0.6-1.9)$ & $1.1(0.6-2.4)$ & $1.2(0.5-2.6)$ & 0.012 \\
\hline LDL, mmol/L & $2.7 \pm 0.8$ & $2.7 \pm 0.8$ & $2.6 \pm 0.9$ & $2.5 \pm 0.8$ & 0.007 \\
\hline $\mathrm{HDL}, \mathrm{mmol} / \mathrm{L}$ & $1.1(0.9-1.3)$ & $1.1(0.9-1.4)$ & $1.1(0.9-1.4)$ & $1.2(0.9-1.4)$ & 0.797 \\
\hline MCV, fL & 89.8 (87.4-92.3) & 88.8 (86.5-92.0) & $89.0(86.7-92.1)$ & $87.8(82.5-90.7)$ & $<0.001$ \\
\hline PDW, \% & $13.7 \pm 2.4$ & $13.9 \pm 2.5$ & $13.9 \pm 2.6$ & $13.9 \pm 2.5$ & 0.475 \\
\hline Platelet, $10^{9} / \mathrm{L}$ & $199.1 \pm 51.4$ & $186.2 \pm 46.9$ & $183.8 \pm 52.4$ & $186.5 \pm 52.2$ & 0.005 \\
\hline
\end{tabular}

RDW quartiles 1 to 4 were defined by $<12.5 \%, 12.5 \%$ to $12.8 \%, 12.9 \%$ to $13.4 \%$, and $>13.4$, respectively. RDW, red blood distribution width; NYHA, New York Heart Association; SBP, systolic blood pressure; DBP, diastolic blood pressure; LVEDD, left ventricular end diastolic dimension; IVST, interventricular septal thickness; LVOT, left ventricular outflow tract; LVEF, left ventricular ejection fraction; Hs-CRP, high sensitive C-reactive protein; eGFR, estimated glomerular filtration rate; LDL, low-density lipoprotein; HDL, high-density lipoprotein; MCV, mean corpuscular volume; PDW, platelet distribution width; BNP, brain natriuretic peptide.

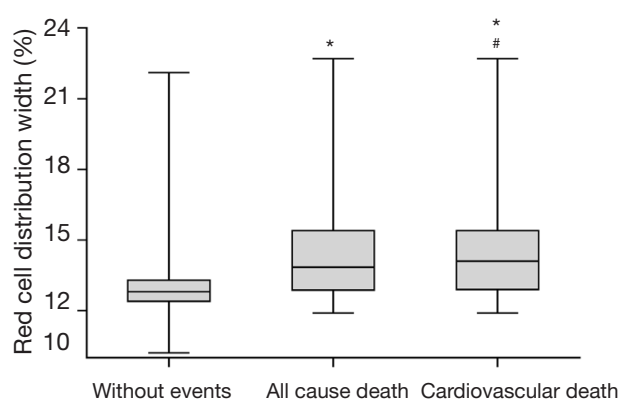

Figure 1 Comparison of red blood distribution width among patients without events, all cause death and cardiovascular death. *, $\mathrm{P}<0.05$, all-cause and cardiovascular death $v$ s. without events;, $\mathrm{P}=$ NS, all-cause death $v s$. cardiovascular death.

[fully adjusted hazards ratio (HR) per RDW-SD increase: $\mathrm{HR}=1.76,95 \%$ CI: 1.54-2.05, $\mathrm{P}<0.001$; per RDW-SD increase $=1.91,95 \%$ confidence interval $(\mathrm{CI}): 1.63-2.22$, respectively; Tables 2,3]. In addition, when participants were divided into four categories according to their baseline quartile of RDW, a graded relation between RDW and clinical outcomes remained. For instance, the fully adjusted risk of mortality and adverse cardiovascular events in the highest quartile of RDW was significantly higher (HR $=2.54,95 \%$ CI: $1.21-5.34$, P for trend $=0.001$; $\mathrm{HR}=2.56$, $95 \%$ CI: $1.27-5.17$, P for trend $=0.002$, respectively) than in the referent group (Table 4).

\section{Discussion}

To the best of our knowledge, the present study is the first to assess the relationship between RDW values and the all-cause and cardiovascular mortality in patients with HOCM who underwent septal myectomy. The main finding of this study was that during the median follow-up period of 32 (IQR, 17-53) months, increased RDW was a strong independent predictor of greater allcause and cardiovascular death in these patients, whether as continuous or categorical variables. This association persisted even after adjustment for clinical and laboratory determinants, such as age, sex, body mass index, and NYHA class.

RDW is a measure of variability in the size of erythrocytes and has been found to be a powerful indicator 


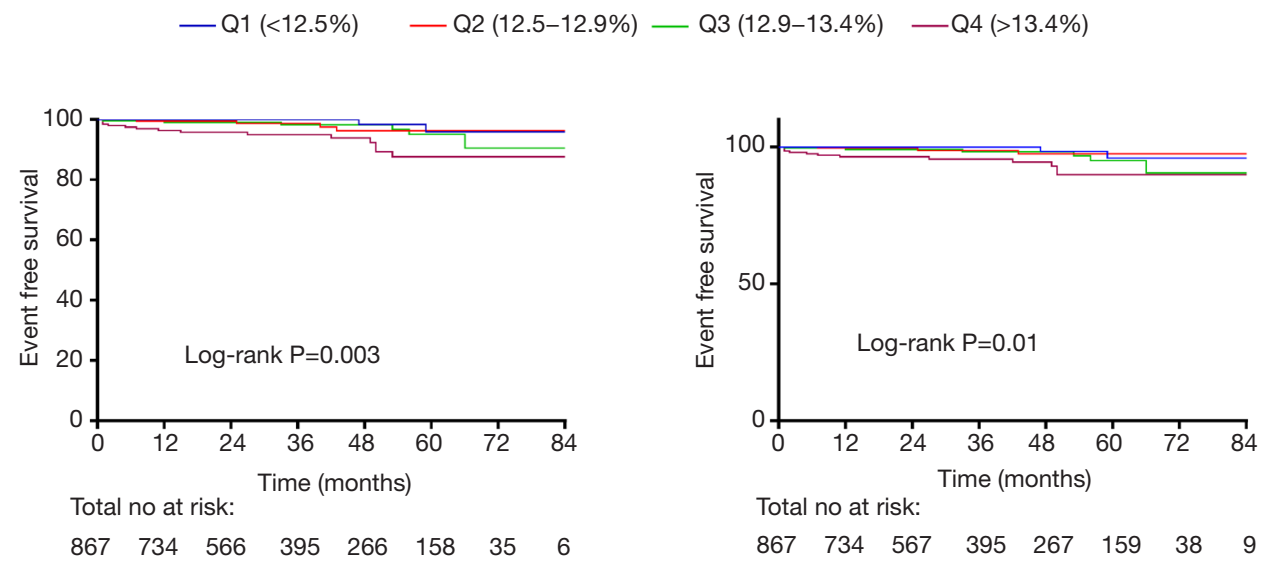

Figure 2 Kaplan-Meier curves stratified per baseline red blood distribution width quartile for the all-cause and cardiovascular death. Q1, first quartile; Q2, second quartile; Q3, third quartile; Q4, fourth quartile.

Table 2 Univariate and multivariate predictors all cause death according to the continuous of RDW

\begin{tabular}{|c|c|c|c|c|}
\hline Variables & \multicolumn{2}{|c|}{ Univariable } & \multicolumn{2}{|c|}{ Multivariate } \\
\hline Age & $1.005(0.97-1.04)$ & 0.780 & - & - \\
\hline Male sex & $0.87(0.40-1.89)$ & 0.717 & - & - \\
\hline Body mass index & $1.09(0.99-1.21)$ & 0.082 & - & - \\
\hline Left atrial diameter & 1.07 (1.02-1.12) & 0.006 & - & - \\
\hline Low density lipoprotein & $0.59(0.37-0.94)$ & 0.026 & - & - \\
\hline Log(NT-pro-BNP) & $4.76(1.38-16.44)$ & 0.014 & - & - \\
\hline Maze procedure & $3.51(1.21-10.20)$ & 0.021 & - & - \\
\hline
\end{tabular}

a , entered into risk factor as continuous variable. RDW, red blood distribution width; HR, hazard ratio; Cl, confidence interval; NYHA, New York Heart Association; BNP, brain natriuretic peptide; SD, standard deviation.

of mortality in other cardiac cohorts, including patients with chronic heart failure $(5,15)$, pulmonary hypertension (8), those undergoing coronary artery bypass grafting (7), and even in the general population (16). Recently, a study revealed that high RDW is an independent predictor of hospitalization for heart failure in patients with HCM (12). No previous reports have been published on the relationship between RDW and HOCM in patients who underwent septal myectomy. In the present study, we found that RDW was significantly associated with adverse clinical outcomes in this population of patients.

The mechanisms linking RDW to morbidity and mortality in patients with HOCM are unclear; however, this may indicate the severity and burden of disease. RDW is elevated in the conditions of ineffective erythropoiesis, such as inflammation, blood transfusion, iron deficiency, malnutrition, inadequate production of erythropoietin, oxidative stress, and impaired renal function (3). In our study, RDW was positively related to hs-CRP, which may reflect the chronic inflammatory state in these patients. 
Table 3 Univariate and multivariate predictors of cardiovascular death according to the continuous of RDW

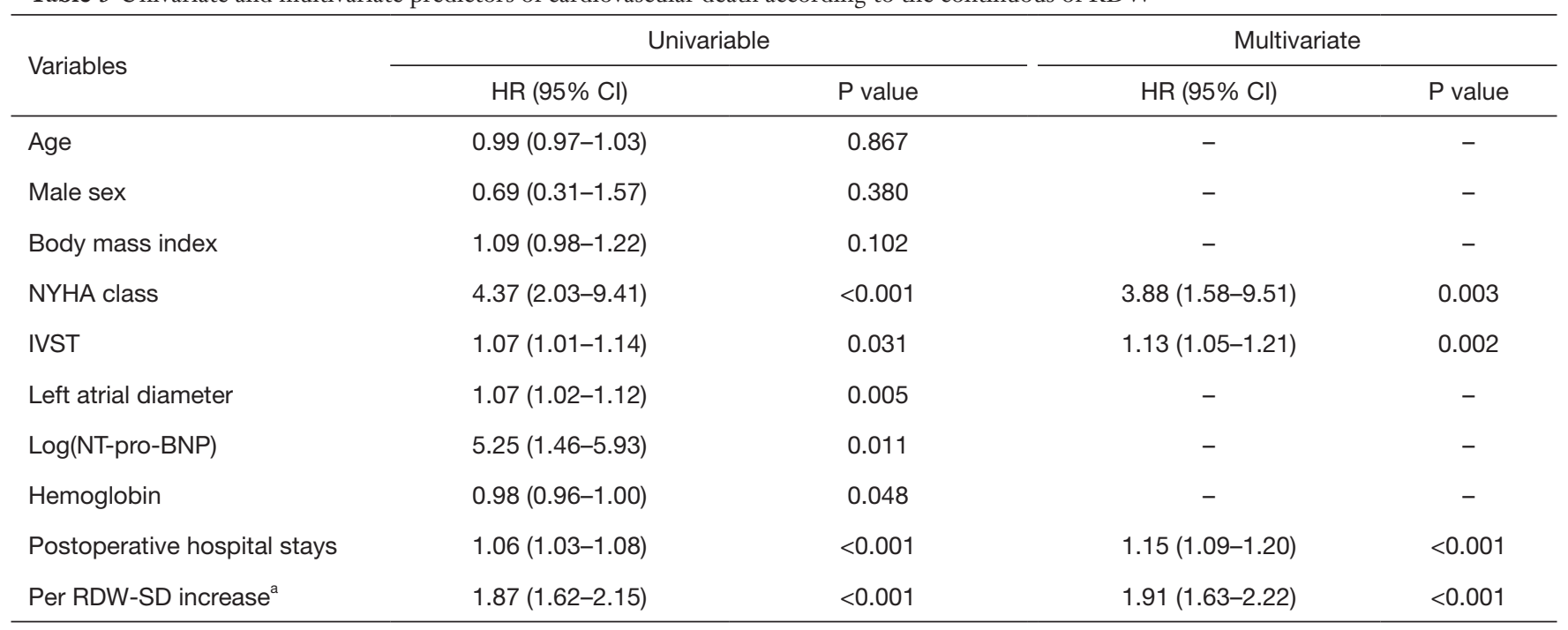

a, entered into risk factor as continuous variable. RDW, red blood cell distribution width; $\mathrm{HR}$, hazard ratio; Cl, confidence interval; NYHA, New York Heart Association; IVST, interventricular septal thickness; BNP, brain natriuretic peptide; SD, standard deviation.

Table 4 Multivariable Cox proportional hazards analysis for predictors of the all cause and cardiovascular death according to baseline RDW quartiles

\begin{tabular}{|c|c|c|c|c|c|}
\hline Variable & Quartile $1(n=246)$ & Quartile $2(n=202)$ & Quartile $3(n=220)$ & Quartile $4(n=246)$ & $P$ for trend ${ }^{a}$ \\
\hline Median of RDW & $12.1 \%$ & $12.6 \%$ & $13.1 \%$ & $14.0 \%$ & \\
\hline No. of cases & 2 & 4 & 6 & 14 & \\
\hline Model 1 & Reference & $2.15(0.39-11.74)$ & $2.67(0.54-13.23)$ & 7.20 (1.63-31.73) & 0.001 \\
\hline Model 3 & Reference & $1.19(0.52-2.72)$ & $0.99(0.44-2.22)$ & $2.54(1.21-5.34)$ & 0.001 \\
\hline \multicolumn{6}{|c|}{ Cardiovascular events } \\
\hline No. cases & 2 & 3 & 6 & 12 & \\
\hline Model 1 & Reference & $1.63(0.27-9.75)$ & $2.70(0.55-13.42)$ & $6.25(1.39-27.96)$ & 0.002 \\
\hline
\end{tabular}

$\mathrm{HR}$ and $95 \% \mathrm{Cl}$ were calculated with the use of Cox proportional hazards regression model. ${ }^{\text {, }}$, calculated by assigning the median level in each quartile to participants and evaluating this variable continuously. Model 1 has not been adjusted. Model 2 adjusted for age, sex, and body mass index. Model 3 additionally was adjusted for NYHA class, left atrial diameter, low density lipoprotein, log(NT-pro-BNP), postoperative hospital stay, maze procedure and hemoglobin. Model 4 additionally was adjusted for log(NT-pro-BNP), IVST, left atrial diameter, hemoglobin and postoperative hospital stay. RDW, red blood distribution width; BNP, brain natriuretic peptide; NYHA, New York Heart Association; IVST, interventricular septal thickness; HR, hazard ratio; CI, confidence interval.

However, multiple Cox analysis revealed no relation between hs-CRP and all-cause death. This was consistent with a previous study's finding that there is no association between RDW and inflammatory cytokines in patients with heart failure (17). In addition, we found that RDW is positively associated with left ventricular outflow tract gradient and mitral valve regurgitation. Increased left ventricular outflow tract gradient may lead to increased 
blood shear stress, leading to increase in RDW (18), and may further increase the severity of mitral valve regurgitation. Previous studies have reported that anemia is associated with left ventricular hypertrophy and adverse cardiovascular events in patients with heart failure (19). However, in the present study, the percentage of anemia did not differ among the different RDW quartiles groups. Furthermore, we found that the prevalence of syncope was significantly higher in patients with higher RDW. A recent meta-analysis has demonstrated that syncope was a significant prognostic factor for cardiovascular death in patients with HCM (20). However, the exact mechanism behind the relationship between RDW and syncope in these patients is unclear; further studies will be required to elucidate this.

There are several mechanisms that might be associated with RDW levels and HOCM patients. First, studies have reported that the renin-angiotensin system is associated with an increased risk in patients with HCM (21). In addition, activation of the renin-angiotensin system is associated with increased erythropoiesis, which could also increase RDW (22). Therefore, one could speculate that a possible link between RDW and HCM lies in the increased angiotensin or adrenergic hormone levels, which could increase RDW and reduce cardiac function. Second, a recent study has shown that iron deficiency has a detrimental impact on the longterm survival of patients with heart failure, which is partially independent of red cell indices (23); this can, in turn, result in left ventricular diastolic dysfunction because iron plays a key role in oxygen metabolism and energy production in cardiomyocytes. In addition, it is also associated with reduced cardiac energetic reserve. Furthermore, iron deficiency is associated with an increase in RDW. Whether these relationships can lead to morbidity and mortality in patients with HOCM after surgery will require further study. Third, the estimated glomerular filtration rate was negatively associated with the increase in RDW in our study. Previous studies have attributed the persistent increase of RDW in cardiovascular diseases to the effective stimulation of erythropoiesis by erythropoietin, a hormone secreted by the kidneys during hypoxic events, which in turn promotes the release of enlarged red blood cells from bone marrow (11). In addition, a recent study has reported that there is a risk of estimated glomerular filtration rate decline in patients with HCM and obstructive sleep apnea (24). These may contribute to adverse clinical outcomes in these patients. Thus, further research is warranted to identify the exact connections between these pathophysiological links.
Therefore, it is important to determine an inexpensive, yet effective, predictor of the long-term results in these patients prior to surgery. RDW is directly available as part of the automated full blood count, a routine component of standard blood testing. The measurement is easy, inexpensive, rapid, does not require specific skills or instrumentation, and is readily available in virtually all clinical laboratories worldwide. The findings of previous studies and our current research demonstrate that RDW should not only be used as an indicator of differential diagnosis of anemia but also used as a potentially effective predictor of long-term prognosis in cardiovascular diseases, including HOCM, following septal myectomy. This readily available biomarker could be considered an additive biomarker for risk stratification in patients with HOCM who underwent septal myectomy.

\section{Limitations}

This study has several limitations. First, our analysis is retrospective and may be only viewed as hypothesisgenerating; prospective data regarding the prognostic value of RDW in patients with HOCM who underwent septal myectomy are required. Second, not all patients in the study underwent cardiac magnetic resonance imaging; therefore, we did not compare the results of RDW with cardiac magnetic resonance imaging data. Third, the study lacked data regarding erythropoietin, folate, vitamin B12, and iron levels, as well as the activation level of renin-angiotensin system; these may have contributed to the mechanisms underlying the association between RDW and clinical outcomes.

\section{Conclusions}

Higher RDW is independently associated with all-cause death and adverse cardiovascular events in patients with HOCM after septal myectomy. This readily available biomarker could be considered an additive biomarker for risk stratification in these patients. However, future research is warranted for further evaluation of the association between RDW and outcomes in patients with HOCM who underwent septal myectomy, in order to improve understanding of the pathophysiology and enhance risk stratification for these patients.

\section{Acknowledgements}

Funding: This work was supported by National Natural 
Science Foundation of China (Grant No. 81570276) and Beijing Postdoctoral Research Foundation.

\section{Footnote}

Reporting Checklist: The authors have completed the STROBE reporting checklist. Available at http://dx.doi. org/10.21037/jtd-20-2528

Data Sharing Statement: Available at http://dx.doi. org/10.21037/jtd-20-2528

Conflicts of Interest: All authors have completed the ICMJE uniform disclosure form (available at http://dx.doi. org/10.21037/jtd-20-2528). The authors have no conflicts of interest to declare.

Ethical Statement: The authors are accountable for all aspects of the work in ensuring that questions related to the accuracy or integrity of any part of the work are appropriately investigated and resolved. The study was conducted in accordance with the Declaration of Helsinki (as revised in 2013). The study was approved by the Ethics Committee of the Fuwai Hospital (Ethic committee study number: 2019-1213). Informed consent was obtained from all patients.

Open Access Statement: This is an Open Access article distributed in accordance with the Creative Commons Attribution-NonCommercial-NoDerivs 4.0 International License (CC BY-NC-ND 4.0), which permits the noncommercial replication and distribution of the article with the strict proviso that no changes or edits are made and the original work is properly cited (including links to both the formal publication through the relevant DOI and the license). See: https://creativecommons.org/licenses/by-nc-nd/4.0/.

\section{References}

1. American College of Cardiology Foundation/American Heart Association Task Force on Practice; American Association for Thoracic Surgery; American Society of Echocardiography, et al 2011 ACCF/AHA guideline for the diagnosis and treatment of hypertrophic cardiomyopathy: a report of the American College of Cardiology Foundation/American Heart Association Task Force on Practice Guidelines. J Thorac Cardiovasc Surg 2011;142:e153-203.
2. O'Hanlon R, Grasso A, Roughton M, et al. Prognostic significance of myocardial fibrosis in hypertrophic cardiomyopathy. J Am Coll Cardiol 2010;56:867-74.

3. Li N, Zhou H, Tang Q. Red Blood Cell Distribution Width: A Novel Predictive Indicator for Cardiovascular and Cerebrovascular Diseases. Dis Markers 2017;2017:7089493.

4. Felker GM, Allen LA, Pocock SJ, et al. Red cell distribution width as a novel prognostic marker in heart failure: data from the CHARM Program and the Duke Databank. J Am Coll Cardiol 2007;50:40-7.

5. van Kimmenade RRJ, Mohammed AA, Uthamalingam $\mathrm{S}$, et al. Red blood cell distribution width and 1-year mortality in acute heart failure. European Journal of Heart Failure 2010;12:129-36.

6. Tonelli M, Sacks F, Arnold M, et al. Relation Between Red Blood Cell Distribution Width and Cardiovascular Event Rate in People With Coronary Disease. Circulation 2008;117:163-8.

7. Warwick R, Mediratta N, Shaw M, et al. Red cell distribution width and coronary artery bypass surgery. Eur J Cardiothorac Surg 2013;43:1165-9.

8. Rhodes CJ, Wharton J, Howard LS, et al. Red cell distribution width outperforms other potential circulating biomarkers in predicting survival in idiopathic pulmonary arterial hypertension. Heart 2011;97:1054-60.

9. Collas VM, Paelinck BP, Rodrigus IE, et al. Red cell distribution width improves the prediction of prognosis after transcatheter aortic valve implantation. Eur J Cardiothorac Surg 2016;49:471-7.

10. Aung N, Dworakowski R, Byrne J, et al. Progressive rise in red cell distribution width is associated with poor outcome after transcatheter aortic valve implantation. Heart 2013;99:1261-6.

11. Danese E, Lippi G, Montagnana M. Red blood cell distribution width and cardiovascular diseases. J Thorac Dis 2015;7:E402-11.

12. Yang HJ, Liu X, Qu C, et al. Usefulness of Red Blood Cell Distribution Width to Predict Heart Failure Hospitalization in Patients with Hypertrophic Cardiomyopathy. Int Heart J 2018;59:779-85.

13. Authors/Task Force m, Elliott PM, Anastasakis A, et al. 2014 ESC Guidelines on diagnosis and management of hypertrophic cardiomyopathy: the Task Force for the Diagnosis and Management of Hypertrophic Cardiomyopathy of the European Society of Cardiology (ESC). Eur Heart J 2014;35:2733-79.

14. Wang S, Cui H, Yu Q, et al. Excision of anomalous muscle 
bundles as an important addition to extended septal myectomy for treatment of left ventricular outflow tract obstruction. J Thorac Cardiovasc Surg 2016;152:461-8.

15. Borné Y, Smith JG, Melander O, et al. Red cell distribution width and risk for first hospitalization due to heart failure: a population-based cohort study. Eur J Heart Fail 2011;13:1355-61.

16. Tajuddin SM, Nalls MA, Zonderman AB, et al. Association of red cell distribution width with all-cause and cardiovascular-specific mortality in African American and white adults: a prospective cohort study. J Transl Med 2017;15:208.

17. Senthong V, Hudec T, Neale S, et al. Relation of Red Cell Distribution Width to Left Ventricular End-Diastolic Pressure and Mortality in Patients With and Without Heart Failure. Am J Cardiol 2017;119:1421-7.

18. Vahidkhah K, Cordasco D, Abbasi M, et al. Flow-Induced Damage to Blood Cells in Aortic Valve Stenosis. Ann Biomed Eng 2016;44:2724-36.

19. Lee WH, Hsu PC, Chu CY, et al. Anemia as an

Cite this article as: Wang S, Song C, Cui H, Zhu C, Wu R, Huang X, Lai Y, Wang S. Red blood cell distribution width is associated with adverse cardiovascular events after septal myectomy. J Thorac Dis 2021;13(2):582-591. doi: 10.21037/jtd20-2528
Independent Predictor of Adverse Cardiac Outcomes in Patients with Atrial Fibrillation. Int J Med Sci 2015;12:618-24.

20. Liu Q, Li D, Berger AE, et al. Survival and prognostic factors in hypertrophic cardiomyopathy: a meta-analysis. Sci Rep 2017;7:11957.

21. Rani B, Kumar A, Bahl A, et al. Renin-angiotensin system gene polymorphisms as potential modifiers of hypertrophic and dilated cardiomyopathy phenotypes. Mol Cell Biochem 2017;427:1-11.

22. Park TS, Zambidis ET. A role for the renin-angiotensin system in hematopoiesis. Haematologica 2009;94:745-7.

23. Tkaczyszyn M, Comín-Colet J, Voors AA, et al. Iron deficiency and red cell indices in patients with heart failure. Eur J Heart Fail 2018;20:114-22.

24. Wang SY, Luo J, Dong YF, et al. Risk of glomerular filtration rate decline in patients with hypertrophic cardiomyopathy and obstructive sleep apnoea. Sci Rep 2017;7:17399. 


\section{Supplementary}

Table S1 Factors associated with higher levels of RDW in multivariable linear regression

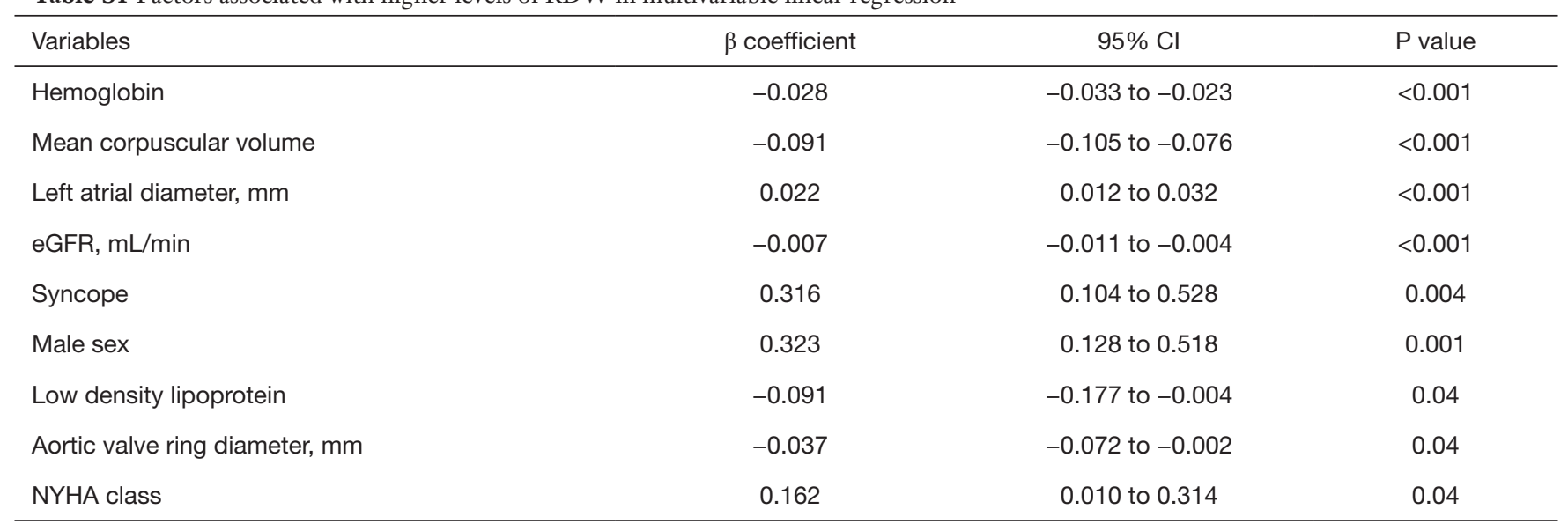

eGFR, estimated glomerular filtration rate; NYHA, New York Heart Association.

Table S2 Perioperative data among different groups

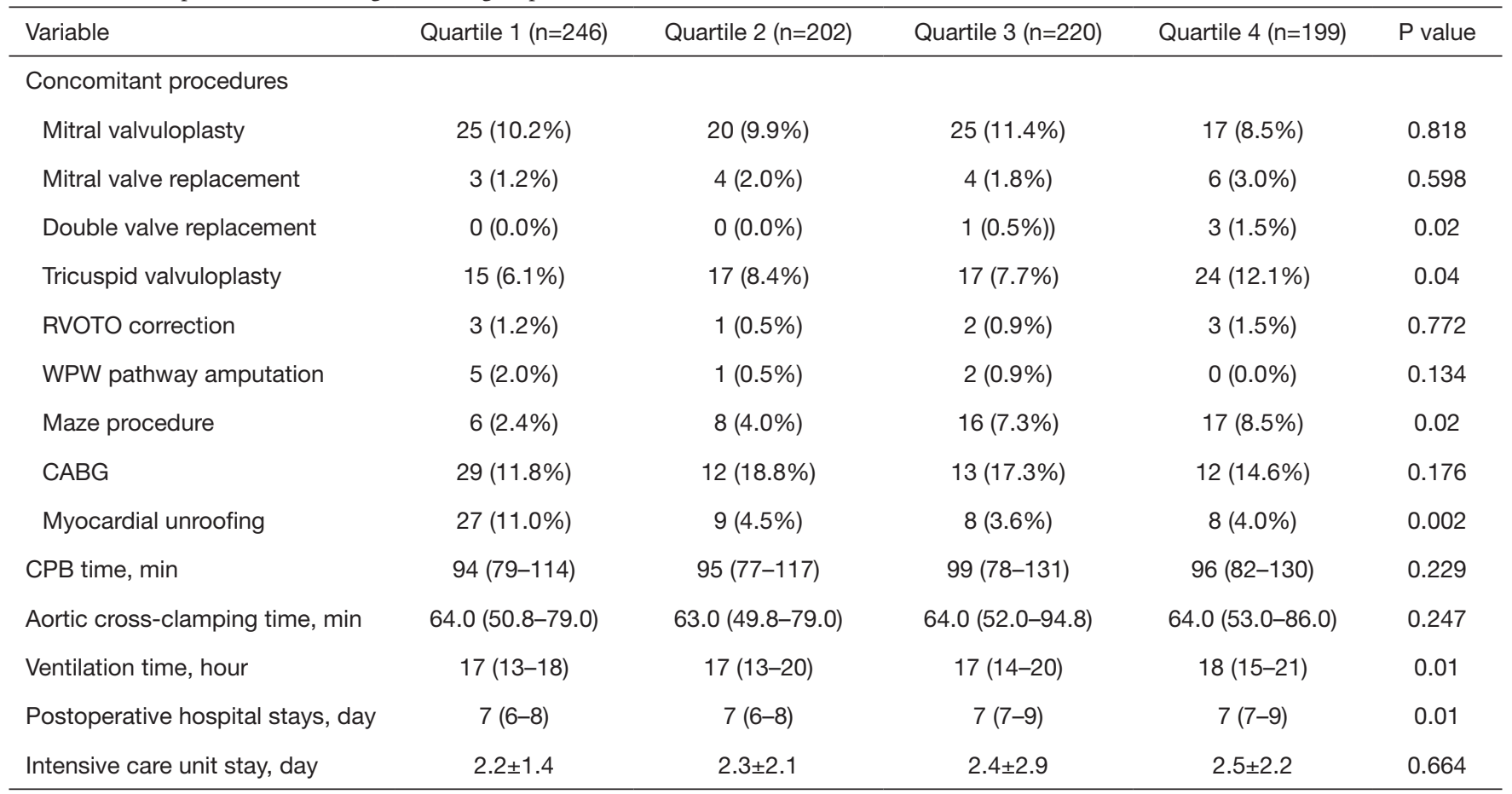

RDW quartiles 1 to 4 were defined by $<12.5 \%, 12.5 \%$ to $12.8 \%, 12.9 \%$ to $13.4 \%$, and $>13.4$, respectively. RVOTO, right ventricular outflow tract obstruction; WPW, Wolff Parkinson White; CPB, cardiopulmonary bypass time; CABG, coronary artery bypass grafting. 